\title{
Excessive daytime sleepiness, nocturnal sleep duration and psychopathology among Nigerian university students
}

\author{
Celestine Okorome Mume, BSc, MB ChB, MSc, FMCPsych \\ Kamildeen Oladimeji Olawale, MB BS \\ Adeagbo Funminiyi Osundina, MB BS
}

Department of Mental Health, Obafemi Awolowo University Teaching

Hospitals Complex, Ile-Ife, Osun State, Nigeria

Background and objectives. Short nocturnal sleep duration resulting in sleep debt may be a cause of excessive daytime sleepiness (EDS). Severity of depression (psychopathology) has been found to be directly related to EDS. There is an association between sleep duration and mental health, so there may therefore be an interrelationship between sleep duration, EDS and psychopathology. The objectives of this study were to determine the prevalence rates of EDS and general psychopathology among university students in Nigeria; determine the range of and mean sleep duration in the students; and determine the extent to which sleep duration and EDS predict general psychopathology in the same group of subjects.

Materials and methods. Eight hundred and forty-five students at Obafemi Awolowo University, lle-Ife, Nigeria, were recruited for the study. The subjects were required to provide information on their age, gender and the total amount of sleep per night they usually had. General psychopathology was assessed using the English language version of the 30-item General Health Questionnaire (GHQ-30). They were also evaluated for EDS using the English language version of the Epworth Sleepiness Scale (ESS).

Results. Six hundred and thirty-four subjects $(75.03 \%$ of the participants) provided complete data. The prevalence of EDS was $11.2 \%$ and the rate of general psychopathology in the subjects $13.1 \%$. The range of sleep duration was 2 - 9 hours with a mean of 5.1 hours (standard deviation 1.3). On a regression model with the GHQ score as the dependent variable and sleep duration and ESS as the independent variables, the correlation coefficient between EDS, sleep duration and psychopathology (R) was 0.47 .

Conclusion. EDS and psychopathology are common in the student population studied. Nocturnal sleep duration for an average student is far less than that for an average adult. Nocturnal sleep duration and EDS acted as moderate predictors of general psychopathology among Nigerian university students.
The average adult human sleeps for about 8 of every 24 hours.' Most of our sleep occurs at night, since we function predominantly during the day and it is necessary to be awake and alert. Under certain conditions, humans are unable to maintain the state of wakefulness needed for daytime functioning. Excessive daytime sleepiness (EDS) manifests with increased sleepiness in situations in which the individual would reasonably be expected to be awake and alert.

Common causes of EDS include sleep deprivation, ${ }^{2}$ obstructive sleep apnoea, narcolepsy, idiopathic hypersomnia and sedative/ hypnotic drugs. ${ }^{3}$ Obstructive sleep apnoea is characterised by snoring and episodes of apnoeic attacks during sleep. Narcolepsy is characterised by excessive daytime sleepiness, cataplexy (sudden decrease or loss of muscle tone often precipitated by intense emotion), sleep paralysis and hypnagogic (sleeponset) hallucinations. The features of idiopathic hypersomnia include chronic sleepiness without cataplexy or other features of narcolepsy.

Short total nocturnal hours of sleep and depression are among the known risk factors for daytime sleepiness. ${ }^{4}$ Total sleep time has an inverse relationship with excessive daytime sleepiness. ${ }^{4}$ On the other hand, the severity of depression (psychopathology) has been found to be directly related to EDS. ${ }^{5-8}$

Many factors account for total nocturnal sleep duration. It has been found that older age, depression, coffee intake, especially at night, and excessive Internet use at night are significantly associated with short nocturnal sleep duration. ${ }^{9}$ Short nocturnal sleep duration has been found to reduce cognitive and behavioural functioning ${ }^{10}$ and to be associated with poor health status. ${ }^{11}$ Short sleep duration also increases the risk of motor vehicle accidents. ${ }^{12}$ It would therefore appear that both daytime sleepiness and sleep duration are important variables contributing to neurocognitive and behavioural functioning, health status (including mental health), and public safety risks.

Information on the possible relationship between excessive daytime sleepiness, sleep duration and general psychopathology in Nigeria is generally inadequate. The present study aimed to provide information in this regard, with respect to university students. The study aimed to determine the extent to which nocturnal sleep duration and EDS act as predictors of general psychopathology among Nigerian university students, the prevalence rates of EDS and general psychopathology, and the range of and mean nocturnal sleep duration in the study subjects. 


\section{Materials and methods Study setting and ethical considerations}

The proposal for this study was approved by the Ethics and Research Committee of the Obafemi Awolowo University Teaching Hospitals Complex, Ile-Ife, Nigeria. The participants were undergraduates of Obafemi Awolowo University, Ile-Ife. Written informed consent was obtained from all who took part in the study. It was a cross-sectional study.

\section{Sampling}

A total of 845 students were recruited into the study. Obafemi Awolowo University has 13 faculties and a student population of about 25 000. Sixty-five students were recruited from each faculty through a multi-stage, stratified sampling technique, aiming for a total of 845 students.

\section{Instruments}

The General Health Questionnaire is a widely used screening instrument. It detects a wide range of psychological disorders and therefore gives a measure of current mental health. It was developed by Goldberg as a screening instrument to identify psychological distress in primary care settings ${ }^{13}$ and has been extensively used and shown to be a valid and reliable instrument across cultures. ${ }^{1-21}$ The questionnaire was initially developed as a 60-item instrument but there are a wide range of shortened versions including the GHQ-30, the GHQ-28, the GHQ-20 and the GHQ-12. The scale asks whether the subject has experienced a particular symptom recently. Each item is rated on a 4-point scale (less than usual, no more than usual, rather more than usual, or much more than usual). The common scoring methods are bimodal (0-0-1-1) and Likert scoring styles (0-12-3). Depending on the scoring method used, the 30-item version therefore gives a score range of 0 - 30 or 0 - 90, with higher scores indicating greater psychopathology. When the 0-0-1-1 scoring system is used, the cut-off point for identifying 'cases' for the GHQ-30 is a score of $\geq 5$. With the Likert scoring system a score in the range of 0 - 24 indicates that the individual is generally free from psychological disturbance. In the present study, in addition to using the bimodal system for case identification, the Likert scoring system was also used since it gives a preferred distribution for regression analysis.

The Epworth Sleepiness Scale (ESS) was developed by Murray Johns in Australia.22 So called because it was developed at the Epworth Hospital in Melbourne, it is a well-validated, 8-item sleep questionnaire. It is a subjective sleepiness scale in which subjects are asked to score the likelihood of falling asleep in eight different situations. The range of scores on the ESS is 0 - 24, with higher scores indicating greater chances or likelihood of sleepiness. A score of 10 but less than 18 is considered sleepy, while a score of 18 or more is considered very sleepy. In the present study, EDS was defined as ESS score $\geq 10$.

\section{Procedure}

The subjects were required to provide information on their age, gender and the usual total amount of sleep they had per night.
General psychopathology was assessed using the English language version of the GHQ-30, while the English language version of the ESS was used to evaluate EDS.

\section{Statistical analysis}

The data were analysed using the Statistical Packages for the Social Sciences, version 16 (SPSS 16.0). Regression analysis was done with GHQ-30 scores as the dependent variable. The independent variables were sleep duration and ESS score.

\section{Results}

Of the 845 subjects who participated in the study, 634 (75.03\%) provided complete data. The rest were excluded from the analysis. The 634 subjects comprised 397 males (62.6\%) and 237 females (37.4\%). The group had an age range of 17 - 31 years with a mean of 22.1 years (standard deviation (SD) 3.4). The range of scores on the ESS was 3 - 17 with a mean of 9.8 (SD 3.2), while the range for sleep duration was 2 - 9 hours with a mean of 5.1 hours (SD 1.3). The mean score on the GHQ-30 using the Likert scoring system was 19.9 (SD 6.6) with a range of $6-38$. With the bimodal system, the range of scores was $3-13$ and the mean was 6.4 (SD 2.1). These results are set out in Table I.

\begin{tabular}{llll}
\multicolumn{4}{l}{ Table I. Age, ESS score, sleep duration and GHQ-30 } \\
\hline Variable & Range & Mean & SD \\
\hline Age (yrs) & $17-31$ & 22.1 & 3.4 \\
ESS score & $3-17$ & 9.8 & 3.2 \\
Sleep duration (h) & $2-9$ & 5.1 & 1.3 \\
GHQ-30 Likert & $6-38$ & 19.9 & 6.6 \\
GHQ- 30 bimodal & $3-13$ & 6.4 & 2.1 \\
\end{tabular}

Forty-three of the 397 males (10.8\%) and 28 of the 237 females (11.8\%) had EDS, defined as ESS score of $\geq 10\left(x^{2}=0.062, \mathrm{df}=1, p=0.8\right)$. A total of 71 out of the 634 subjects (11.2\%) therefore had EDS. Fiftyone of the 397 males (12.8\%) and 32 of the 237 females (13.5\%) had psychopathology, defined a as GHQ-30 score $\geq 5$ using the bimodal scoring system $\left(x^{2}=0.013, d f=1, p=0.9\right)$. A total of 83 out of the 634 subjects (13.1\%) were therefore identified as cases.

Using a regression model with the GHQ score as the dependent variable and sleep duration and ESS as the independent variables, the correlation coefficients were as follows: the correlation between EDS and psychopathology ( $r 1)$ was $0.41(p=0.000)$, that between sleep duration and psychopathology $(r 2)$ was $-0.36(p=0.00)$, and that between EDS and sleep duration ( $r 3$ ) was -0.37 ( $p=0.000)$. These showed that EDS had a moderate, significant direct relationship with psychopathology; sleep duration had a moderate, significant inverse relationship with psychopathology; and EDS had a moderate, significant inverse relationship with sleep duration. The correlation between EDS, sleep duration and psychopathology (R) was $0.47(p=0.000)$ and $R^{2}$ was 0.22 , showing that EDS and sleep duration accounted for $22 \%$ of the variance of psychopathology by 
virtue of their linear relationship. Nocturnal sleep duration and EDS therefore acted as moderate predictors of general psychopathology among Nigerian university students. The results of the regression analysis are shown in Table II.

\begin{tabular}{|ll|}
\hline $\begin{array}{l}\text { Table II. Regression analysis with psychopathology as dependent vari- } \\
\text { able and ESS and sleep duration as independent variables }\end{array}$ \\
\hline Variable & Value \\
\hline Correlation & \\
$r 1$ & 0.41 \\
$r 2$ & -0.36 \\
$r 3$ & -0.37 \\
Significance $(r 1, r 2, r 3)$ & 0.000 \\
Model summary & \\
R & 0.47 \\
$R^{2}$ & 0.22 \\
Adjusted $R^{2}$ & 0.22 \\
ANOVA & \\
df & 2,631 \\
F & 88.32 \\
Significance & 0.000 \\
The correlation coefficient between EDS and psychopathology is denoted by $r 1$, that between \\
sleep duration and psychopathology by 2 , and that between EDS and sleep duration by r3. The \\
correlation coefficient between EDS, sleep duration and psychopathology is $R$. \\
\hline
\end{tabular}

\section{Discussion}

Many university students are adolescents and young adults, and due to the demands of the academic environment may be involved in late-night study and use of the Internet. They are likely to consume stimulants such as caffeine in order to stay awake and alert at night, a practice found to be common among adolescents. ${ }^{23}$ Their sleep patterns are therefore likely to differ from those of non-students of the same age. ${ }^{6}$ It is therefore not surprising that the mean sleep duration for our subjects was 5.1 hours $(S D=1.3)$, with a range of 2 - 9 hours. However, the present study did not focus on drug use, or aim to determine the causes of short nocturnal sleep duration.

EDS is common, and said to be one of the common complaints of patients seen in sleep clinics. ${ }^{24}$ Its prevalence varies with the study population and operational definition. It is therefore difficult to compare the results of different studies. Prevalence rates of 12 $20 \%$ have been reported in population-based studies. ${ }^{24,25}$ However, prevalence rates of $25.4 \%{ }^{26}$ and $33.3-39.2 \% \%^{27}$ have been reported in schoolchildren. It has also been found that children whose school day started at 07:10 complained significantly more about daytime sleepiness compared with those who started later. ${ }^{28}$ The prevalence of $11.2 \%$ reported in this study is consistent with previous findings in population-based studies. In a recent study, $44.8 \%$ of depressed patients had EDS, defined as ESS score of equal to or greater than $10 .{ }^{8}$

Using the 12-item version of the $\mathrm{GHQ}$, Nur et al. found $27.8 \%$ of medical students to be suffering from an emotional disorder, ${ }^{29}$ while
Radovanovic et al. ${ }^{30}$ identified $14.1 \%$ of medical students as cases using the 60 -item version. The $13.1 \%$ prevalence rate found in the present study is probably a fair representation of the situation in a student population, because the two studies cited ${ }^{29,30}$ focused on medical students, who are likely to have a higher workload than most other students. Students with a heavy workload are more prone to psychopathology than those who have fewer academic demands.

Reduced sleep at night is a known risk factor for EDS. ${ }^{25,31}$ Subjects suffering from EDS may exhibit psychosocial distress. ${ }^{24}$ Psychopathology has been reported to be common among university students, 32,33 though these were specific cases of depression. A previous study has found an association between sleep duration and mental health. ${ }^{23}$ In the present study, an inverse relationship was found between sleep duration and psychopathology, and a direct one was found between psychopathology and EDS.

The present study found an interrelationship between sleep duration at night, EDS and general psychopathology. Although causation may not be easy to establish in this relationship, it is clear that EDS may result from short nocturnal sleep duration due to any cause such as insomnia or sleep deprivation, as well as from psychopathology. This study did not consider possible causes of EDS, psychopathology or short sleep duration, but it is considered that the sleep habits of the students must have been influenced by their academic commitments.

EDS is very disruptive to the lives of individuals, and so is psychopathology - both impair social and occupational functions. Insomnia and short nocturnal sleep duration are regular accompaniments of psychopathology. The triad of EDS, short nocturnal sleep duration and psychopathology must necessarily impact adversely on the lives and academic performance of those who suffer from them. If a student (or indeed any person) is found to be suffering from one of them, it would be beneficial to enquire about the others. Further studies are necessary to establish the exact nature of the predominant relationship between these three variables in student populations. On an individual basis it is sometimes possible to determine when one factor results from another (for example when psychopathology is the cause of insomnia, or when EDS results from sleep deprivation). Appropriate measures are advocated in such cases.

\footnotetext{
References

1. Roth T, Roehrs T. Sleep organization and regulation. Neurology 2000;54 (suppl 1): S2-S7.

2. Pagel JF. Excessive daytime sleepiness. Am Fam Physician 2009;79:391-396.

3. Edd EM, Flores S. Sleepiness or excessive daytime somnolence. Geriatr Nurs 2009:30:53-60.

4. Roehrs TA, Carskadon MA, Dement WC, Roth T. Daytime sleepiness and alertness. In: Kryger MH, Roth T and Dement WC, eds. Principles and Practice of Sleep Medicine. 3rd ed. Philadelphia: Saunders, 2000:43-52.

5. Lundt L. Use of the Epworth sleepiness scale to evaluate the symptom of excessive sleepiness in major depressive disorder. Gen Hosp Psychiatry 2005;27(2):146-148.

6. Hublin C, Kapiro J, Partinen M, Heikkila K, Koskenvuo M. Daytime sleepiness in an adult Finnish population. J Intern Med 1996;239:417-423.

7. Shin K, Yi H, Kim J, Shin C. Prevalence and associated factors of excessive daytime sleepiness in adults. Taehan Kanho Hakhoe Chi 2006;36:829-836.
} 
8. Mume CO. Excessive daytime sleepiness among depressed patients. Libyan J Med 2010;5:4626. DOI: 10.4176/091024.

9. Yen $\mathrm{CF}$, $\mathrm{Ko} \mathrm{CH}$, Yen JY, Cheng CP. The multidimensional correlates associated with short nocturnal sleep duration and subjective insomnia among Taiwanese adolescents. Sleep 2008;31:1515-1525.

10. Maquet P. The role of sleep in learning and memory. Science 2001;294:1048-1052.

11. Chen MY. Adequate sleep among adolescents is positively associated with health status and health-related behaviors. BMC Public Health 2006;6:59

12. Carskadon MA. Regulation of adolescent sleep: implications for behavior. Ann NY Acad Sc 2004;1021:276-291.

13. Goldberg DP, Blackwell B. Psychiatric illness in general practice. A detailed study using a new method of case identification. BMJ 1970;1:439-443.

14. Jacobsen BK, Hasvold T, Hoyer G, Hansen V. The General Health Questionnaire: How many items are really necessary in population surveys? Psychol Med 1995;25:957-962.

15. Ohta Y, Kawasaki N, Araki K, Mine M, Honda S. The factor structure of the General Health Questionnaire (GHQ-30) in Japanese middle-aged and elderly residents. Int J Soc Psychiatry 1995;41:268-275.

16. Jacob KS, Bhugra D, Mann AH. The validation of the 12-item General Health Questionnaire in ethnic Indian women living in the United Kingdom. Psychol Med 1997;27:1215-1217.

17. Kihç C, Rezaki1 M, Rezaki B, et al. General Health Questionnaire (GHQ12 \& GHQ28) psychometric properties and factor structure of the scales in a Turkish primary care sample. Soc Psychiatry Psychiatr Epidemiol 1997;32:327-331

18. Schmitz N, Kruse J, Tress W. Psychometric properties of the General Health Questionnaire (GHQ)-12 in a German primary care. Acta Psychiatr Scand 1999;100:462-468.

19. Pevalin DJ. Multiple applications of the GHQ-12 in a general population sample: an investigation of long-term retest effects. Soc Psychiatry Psychiatr Epidemiol 2000;35:508512.

20. Donath S. The validity of the 12-item General Health Questionnaire in Australia: a comparison between three scoring methods. Aust N Z J Psychiatry 2001;35:231-235.
21. O'Rourke S, MacHale S, Signorini D, Dennis M. Detecting psychiatric morbidity after stroke: Comparison of the GHQ and the HAD Scale. Stroke 1998;29:980-985

22. Johns MW. A new method for measuring daytime sleepiness: the Epworth sleepiness scale. Sleep 1991;14:540-545.

23. Ichikawa K, Matsui T. Tsunoda T, et al. The relationships of sleep duration and mental health with electrocardiographic findings: a retrospective-cohort study in Okinawa, Japan. Environ Health Prev Med 2008;13:227-233.

24. Roth T, Roehrs TA. Etiologies and sequelae of excessive daytime sleepiness. Clin Ther 1996;18:562-576.

25. Pagel JF. Excessive daytime sleepiness. Am Fam Physician 2009;79:391-396.

26. Ng EP, Ng DK, Chan CH. Sleep duration, wake/sleep symptoms, and academic performance in Hong Kong secondary school children. Sleep Breath 2009;13:357-367.

27. Ohida T, Osaki Y, Doi Y, et al. An epidemiologic study of self-reported sleep problems among Japanese adolescents. Sleep 2004;27:978-985.

28. Epstein R, Chillag N, Lavie P. Starting times of school: effects on daytime functioning of fifthgrade children in Israel. Sleep 1998;21(3):250-256.

29. Nur N, Cetinkaya S, Sabanciogullari V, Ozsahin SL, Kavakci O. Emotional disorders among Turkish undergraduate medical students. Pak J Med Sci 2008;24:792-797.

30. Radovanovic Z, Eric LJ, Jevremovic I. The effect of re-testing on the validity of the Genera Health Questionnaire. Soc Psychiatry Psychiatr Epidemiol 1988;23:36-38.

31. Calamaro CJ, Mason TB, Ratcliffe SJ. Adolescents living the 24/7 lifestyle: effects of caffeine and technology on sleep duration and daytime functioning. Pediatrics 2009;123:e10051010.

32. Adewuya OA, Ola BA, Aloba OO, Mapayi BM, Oginni OO. Depression amongst Nigerian university students: Prevalence and sociodemographic correlates. Soc Psychiatry Psychiatr Epidemiol 2006;41:674-678.

33. Khawaja NG, Duncanson K. Using the University Student Depression Inventory to investigate the effect of demographic variables on students' depression. Australian Journal of Guidance and Counselling 2008;18:195-209.

\section{Philosophy \& Ethics}

Two-way connections with mental health

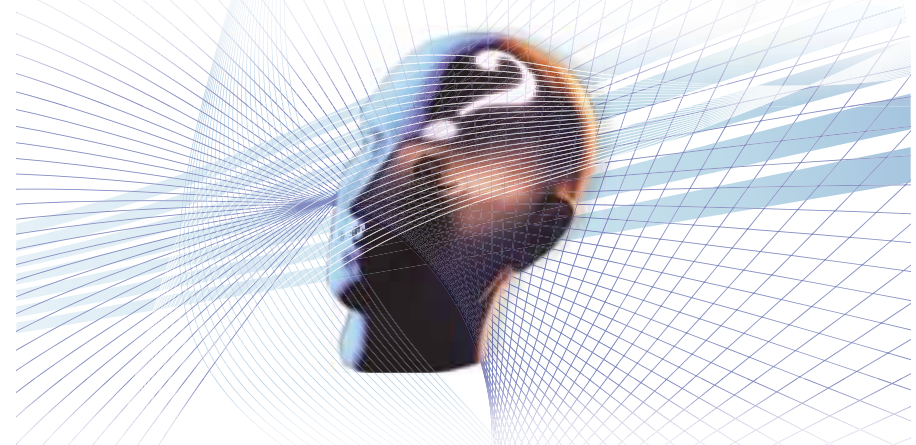

The University of Pretoria, one of South Africa's foremost research institutions, now offers a Master's course (MPhil) in Philosophy \& Ethics of Mental Health in the Department of Psychiatry, Faculty of Health Sciences.

offers philosophical skills and

The course offers seminars, e-learning, case studies and guided writing of a dissertation. Candidates are offered a wide choice of content, as well as publication and $\mathrm{PhD}$ opportunities.

- Mental health practitioners

Philosophers

For more information,

visit www.up.ac.za/pemh

or email the course director,

Prof. Werdie van Staden at

werdie.vanstaden@up.ac.za 